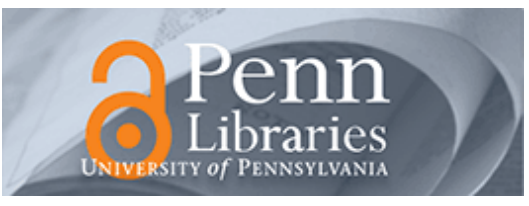

University of Pennsylvania

ScholarlyCommons

$6-27-2012$

\title{
Measurement of Scintillation Efficiency for Nuclear Recoils in Liquid Argon
}

\author{
Dan Gastler \\ Boston University \\ Ed Kearns \\ Boston University \\ Andrew Hime \\ Los Alamos National Laboratory \\ Laura C. Stonehill \\ Los Alamos National Laboratory \\ Stan Seibert \\ Los Alamos National Laboratory
}

See next page for additional authors

Follow this and additional works at: https://repository.upenn.edu/physics_papers

Part of the Physics Commons

\section{Recommended Citation}

Gastler, D., Kearns, E., Hime, A., Stonehill, L. C., Seibert, S., Klein, J., Lippincott, W. H., McKinsey, D. N., \& Nikkel, J. A. (2012). Measurement of Scintillation Efficiency for Nuclear Recoils in Liquid Argon. Retrieved from https://repository.upenn.edu/physics_papers/247

Gastler, D., Kearns, E., Hime, A., Stonehill, L. C., Seibert, S., Klein, J., Lippincott, W. H., McKinsey, D. N., \& Nikkel, J. A. (2012). Measurement of Scintillation Efficiency for Nuclear Recoils in Liquid Argon. Physical Review C, 85(6), 065811. doi: 10.1103/PhysRevC.85.065811

(c) 2012 American Physical Society

This paper is posted at ScholarlyCommons. https://repository.upenn.edu/physics_papers/247

For more information, please contact repository@pobox.upenn.edu. 


\title{
Measurement of Scintillation Efficiency for Nuclear Recoils in Liquid Argon
}

\author{
Abstract \\ The scintillation light yield of liquid argon from nuclear recoils relative to electronic recoils has been \\ measured as a function of recoil energy from $10 \mathrm{keVr}$ up to $250 \mathrm{keVr}$ at zero electric field. The scintillation \\ efficiency, defined as the ratio of the nuclear recoil scintillation response to the electronic recoil response, \\ is $0.25 \pm 0.01+0.01$ (correlated) above $20 \mathrm{keVr}$. \\ Disciplines \\ Physical Sciences and Mathematics | Physics

\section{Comments} \\ Gastler, D., Kearns, E., Hime, A., Stonehill, L. C., Seibert, S., Klein, J., Lippincott, W. H., McKinsey, D. N., \& \\ Nikkel, J. A. (2012). Measurement of Scintillation Efficiency for Nuclear Recoils in Liquid Argon. Physical \\ Review C, 85(6), 065811. doi: 10.1103/PhysRevC.85.065811 \\ (c) 2012 American Physical Society

\section{Author(s)} \\ Dan Gastler, Ed Kearns, Andrew Hime, Laura C. Stonehill, Stan Seibert, Josh Klein, W. Hugh Lippincott, \\ Daniel N. McKinsey, and James A. Nikkel
}




\title{
Measurement of scintillation efficiency for nuclear recoils in liquid argon
}

\author{
Dan Gastler, ${ }^{1}$ Ed Kearns, ${ }^{1}$ Andrew Hime, ${ }^{2}$ Laura C. Stonehill, ${ }^{2}$ Stan Seibert, ${ }^{2}$ Josh Klein, ${ }^{3}$ W. Hugh Lippincott, ${ }^{4}$ \\ Daniel N. McKinsey, ${ }^{4}$ and James A. Nikkel ${ }^{4}$ \\ ${ }^{1}$ Boston University, Boston, Massachusetts 02215, USA \\ ${ }^{2}$ Los Alamos National Laboratory, Los Alamos, New Mexico 87545, USA \\ ${ }^{3}$ University of Pennsylvania, Philadelphia, Pennsylvania 19104, USA \\ ${ }^{4}$ Yale University, New Haven, Connecticut 06511, USA
}

(Received 5 April 2010; revised manuscript received 8 May 2012; published 27 June 2012)

\begin{abstract}
The scintillation light yield of liquid argon from nuclear recoils relative to electronic recoils has been measured as a function of recoil energy from $10 \mathrm{keVr}$ up to $250 \mathrm{keVr}$ at zero electric field. The scintillation efficiency, defined as the ratio of the nuclear recoil scintillation response to the electronic recoil response, is $0.25 \pm 0.01+0.01$ (correlated) above $20 \mathrm{keVr}$.
\end{abstract}

DOI: 10.1103/PhysRevC.85.065811

PACS number(s): 29.40.Mc, 95.35.+d

\section{INTRODUCTION}

A number of existing and proposed experiments use liquefied noble gases as detection media for weakly interacting massive particles (WIMPs) [1-5], a well motivated dark-matter candidate [6]. Liquefied noble gases have a high scintillation yield, are relatively simple to purify of both radioactive contaminants and light absorbers, and should be easily scalable to the large masses required for very sensitive detectors. Although the best limit for the spin-independent WIMPnucleon cross section is currently set by the germanium-based CDMS experiment [7] at $3.8 \times 10^{-44} \mathrm{~cm}^{2}$ for a $70-\mathrm{GeV}$ WIMP mass, the XENON-10 experiment has set a comparable limit of $8.8 \times 10^{-44} \mathrm{~cm}^{2}$ for a 100-GeV WIMP mass [8], showing that liquefied noble gases are viable dark-matter targets.

Events in a noble liquid dark-matter detector may arise from scattering off of the nucleus or atomic electrons; dark matter will only scatter off the nucleus to an appreciable extent. The ratio of the scintillation light yield for nuclear recoil events relative to electronic recoil events is defined as the scintillation efficiency or $L_{\text {eff }}$.

A WIMP dark-matter search requires an energy threshold on the order of tens of $\mathrm{keV}$, and it is necessary to measure the scintillation efficiency down to this energy threshold so as to quantify the WIMP detection sensitivity. To make this measurement of $L_{\text {eff }}$, a deuterium-deuterium (D-D) neutron generator was used to produce neutrons that scattered from a liquid-argon detector into an organic liquid-scintillator detector used as a coincidence trigger. The organic scintillator was placed at a series of known angles, and the energies of the selected nuclear recoils in the liquid argon were kinematically determined. The scintillation efficiency was determined from the ratio of the measured electron-equivalent recoil energy at a given scattering angle to the expected nuclear recoil energy $(\mathrm{keVr})$ at that angle. Details of this measurement in a 4-kg liquid argon detector are presented in this paper, along with scintillation efficiency results for nuclear recoil energies between 10 and $250 \mathrm{keVr}$ at zero electric field.

\section{REVIEW OF PHYSICAL PROCESSES AND MEASUREMENTS}

Discrimination between nuclear recoil events that characterize a WIMP signal and electronic recoil events that characterize the primary backgrounds is essential in WIMP detectors, particularly for the case of liquid argon which contains the radioactive isotope ${ }^{39} \mathrm{Ar}$. The noble liquid detectors use two methods to achieve this discrimination. Single-phase detectors use pulse-shape discrimination (PSD) based solely on scintillation light to discriminate between event types, while dual-phase detectors can collect both scintillation light and ionization, employing a combination of PSD and the relative size of the light and ionization channels to identify events. PSD is made possible because ionizing radiation in liquid noble gases results in the formation of excited diatomic molecules (excimers) that can exist in either singlet or triplet states, with very different lifetimes. In liquid argon these lifetimes are $7 \mathrm{~ns}$ and $1.5 \mu \mathrm{s}$, respectively $[9,10]$, and the scintillation light is produced in the decay of these states. As different types of excitation produce different ratios of triplet to singlet molecules, the relative amplitudes of the fast and slow components can be used to determine what type of excitation occurred. The effectiveness of this PSD is directly dependent on the number of detected photoelectrons in an event, and thus the light yield for both nuclear recoils and electronic recoils sets the energy threshold for which electronic recoil backgrounds are negligible, in turn determining the ultimate sensitivity of the detector to dark-matter-induced nuclear recoils.

The excimers that provide the scintillation light are formed in two ways. An excited atom (exciton) can combine with another atom in the liquid to produce the excimer, or an ionized atom can combine with another atom to form a diatomic ion, which in turn recombines with an electron, eventually resulting in the production of the excimer. The ratio of exciton production to ion pair production in liquid argon has been calculated to be 0.21 [11], indicating that the majority of the scintillation light in liquid argon comes from excimers formed indirectly from argon ions, rather than directly from excited argon. The average energy required to produce an 
electron-ion pair in liquid argon has been measured to be $23.6 \pm 0.3 \mathrm{eV}[12]$, and the average energy needed to produce a single photon has been calculated to be $19.5 \pm 1.0 \mathrm{eV}$ [13]. From this, the maximum possible scintillation yield in liquid argon is about 51 photons per $\mathrm{keV}$ of deposited energy, in the extreme case where the excimer formation and scintillation processes are perfectly efficient.

In actuality, the absolute light yield is reduced through a number of different mechanisms. Energy may be lost by means other than exciton and ion pair formation, the excitons may undergo nonradiative collisions, and the recombination of diatomic ions may be incomplete. The first mechanism is known to be significant for nuclear recoils, for which a significant portion of the energy is lost to atomic motion, as described by Lindhard et al. [14]. Thus, the scintillation light yield is expected to be reduced for nuclear recoil events compared to electronic recoils. Measurements of the scintillation efficiency for nuclear recoils relative to electronic recoils in liquid xenon [15-19] indicate that there is an additional reduction in the nuclear recoil scintillation yield owing to collisions between free excitons that result in an ion and a ground-state atom, as described by Hitachi [20]. The rate of these biexcitonic collisions is dependent on the density of the excitations; thus, the amount of quenching increases with increasing linear-energy-transfer (LET) and is most significant at larger recoil energies. This mechanism of biexcitonic quenching is expected to apply to liquid argon as well as liquid xenon, and a model for scintillation efficiency in argon, neon, and xenon taking LET into account has been proposed by Mei et al. [21]. A further reduction in scintillation yield can result when some fraction of the ion-electron pairs do not recombine to produce an excimer and the electrons escape instead [13].

Relative scintillation efficiencies in liquid argon have been measured for a number of different particle types. For heavy fission fragments with kinetic energy around $80 \mathrm{MeV}$, the scintillation efficiency relative to $1-\mathrm{MeV}$ electrons has been measured to be $0.21 \pm 0.04$ [22]. For $\alpha$ particles, the scintillation efficiency has been measured to be 0.9 for $6-\mathrm{MeV}$ $\alpha$ 's relative to $1-\mathrm{MeV}$ electrons [22] and 0.4 for $5.3-\mathrm{MeV}$ $\alpha$ 's relative to $1.2-\mathrm{MeV}$ electrons [23]. The observed triplet lifetime from the $5.3-\mathrm{MeV} \alpha$ 's was $800 \mathrm{~ns}$, which may indicate additional absorption owing to impurities, but both values are plausible given the expectation that the scintillation efficiency for $\alpha$ 's should fall somewhere between that of fission fragments and unity. A previous measurement of scintillation efficiency for nuclear recoils in liquid argon by the WARP collaboration gives $0.28 \% \pm 10 \%$, measured at $65 \mathrm{keV}$ average recoil energy [3].

\section{EXPERIMENTAL DETAILS}

The liquid argon scintillation efficiency was measured using the MicroCLEAN detector at Yale University. The active volume is $3.14 \mathrm{~L}$ of liquid argon viewed by two photomultiplier tubes (PMTs). Figure 1 shows a schematic of the central region and PMTs. The active region is defined by a Teflon cylinder $200 \mathrm{~mm}$ in diameter and $100 \mathrm{~mm}$ in height, with

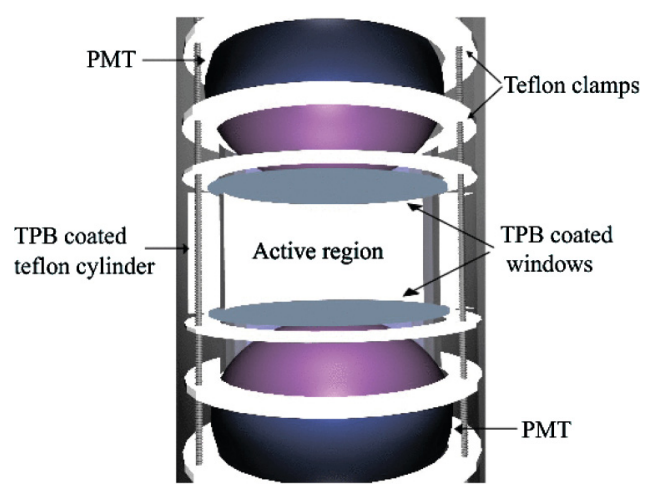

FIG. 1. (Color online) Schematic representation of the scintillation volume and PMT orientation.

two 3-mm-thick fused-silica windows closing the top and the bottom. Two 200-mm-diameter Hamamatsu R5912-02MOD PMTs are held in place by Teflon rings above and below the central volume and view the active region through the windows. Because liquid argon scintillates in the vacuum ultraviolet at $128 \mathrm{~nm}$ [24], all inner surfaces of the Teflon and fused silica are coated with a thin film of tetraphenyl butadiene (TPB) [25]. The TPB shifts the wavelength of the ultraviolet light to approximately $440 \mathrm{~nm}$ so that it may pass through the windows and be detected by the PMTs. Both windows are coated with $0.20 \pm 0.01 \mathrm{mg} / \mathrm{cm}^{2}$ of TPB and the Teflon cylinder is coated with $0.30 \pm 0.01 \mathrm{mg} / \mathrm{cm}^{2}$ of TPB. The Teflon cylinder, windows and PMTs are all immersed directly in liquid argon, contained within a $25-\mathrm{cm}$-diameter by 91-cm-tall stainless-steel vessel.

The stainless steel vessel is housed inside a vacuum dewar, and liquid argon is introduced though a tube on the top of the vessel. The argon is liquefied from purified gas in a copper vessel mounted to the end of a Cryomech PT805 pulse-tube refrigerator. All components that come into contact with the gas or liquid are baked to at least $60^{\circ} \mathrm{C}$, and the ultra-high-purity argon gas (99.999\%) is passed through a heated Omni Nupure III gas-purification getter before entering the vessel. Outgassing can cause impurities to build up in the detector, decreasing the light yield by quenching the argon excimers or absorbing the UV scintillation photons. To avoid signal degradation, the argon is continually circulated through the getter and reliquefied at a rate greater than 2.0 standard liters per minute. No reduction in signal was observed during the run. PMT signals were ditigized using an 8-bit 500 MSPS waveform digitizer with each of the PMTs capturing both low-gain and high-gain waveforms. More details about the experimental apparatus, data acquisition, and purity measurements are available in [10].

A sample oscilloscope trace from an electronic recoil scintillation event in argon is shown in Fig. 2. A 10- $\mu \mathrm{Ci}$ sealed ${ }^{57} \mathrm{Co}$ source is used for daily measurements of the scintillation light yield for electronic recoils, with a sample spectrum shown in Fig. 3 along with results from the simulation to be discussed in the next section. This source produces 122-, $137-$, and $14.4-\mathrm{keV} \gamma$ rays with branching ratios of $86 \%$, $11 \%$, and $9 \%$, respectively. Spectra were taken for each day of data taking, with a Gaussian fit to the 122-keV peak 


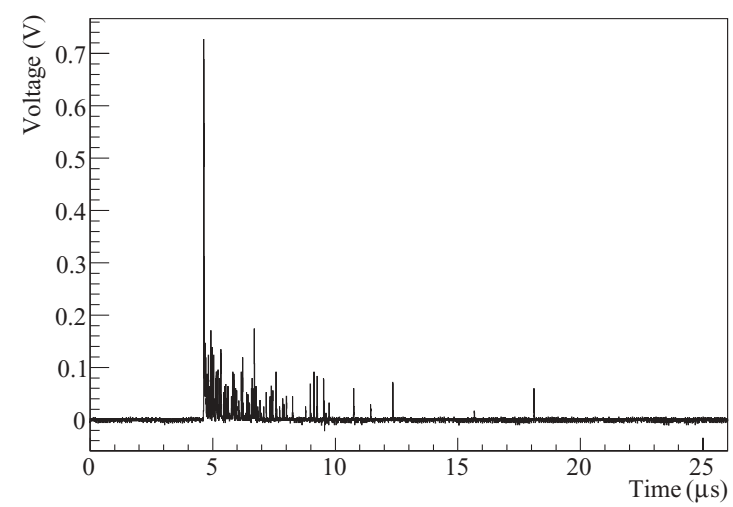

FIG. 2. Example of an electronic recoil-induced scintillation event in liquid argon.

providing a scintillation signal yield calibration for that day in units of photoelectrons per $\mathrm{keV}$ of energy deposited by an electronic recoil, denoted as photoelectrons per keV electron equivalent (keVee). Over the course of the 4-month run, the signal yield remained stable to within $5 \%$ at $4.85 \pm 0.01$ photoelectrons $/ \mathrm{keVee}$. To check the quality of the energy calibration, a $10-\mu \mathrm{Ci}^{22} \mathrm{Na}$ source that produces $511-\mathrm{keV} \gamma$ rays is used as a second point of reference, and the $511-\mathrm{keV}$ line appears at a photoelectron yield that is within $1 \%$ of the value predicted from ${ }^{57} \mathrm{Co}$ source calibration.

A portable Thermo Electron MP320 D-D neutron generator is used as a neutron source, with an organic scintillator detector as a secondary coincidence trigger. The experimental setup can be seen schematically in Fig. 4. In the forward direction, the $\mathrm{D}-\mathrm{D}$ generator produces $2.8 \mathrm{-MeV}$ neutrons. Some of these neutrons scatter in the liquid argon, and for a given position of the organic scintillator, only neutrons that scatter at a specific angle are selected by the coincidence trigger. By changing the angle the organic scintillator makes with the neutron generatorliquid argon detector axis, the scattering energy of the recoil nucleus in the liquid argon can be varied according to the following equation:

$$
\begin{aligned}
E_{\mathrm{rec}}= & \frac{2 m_{n} E_{\mathrm{in}}}{\left(m_{n}+m_{\mathrm{Ar}}\right)^{2}}\left[m_{n}+m_{\mathrm{Ar}}-m_{n} \cos ^{2}(\theta)\right. \\
& \left.-\cos (\theta) \sqrt{m_{\mathrm{Ar}}^{2}+m_{n}^{2} \cos ^{2}(\theta)-m_{n}^{2}}\right]
\end{aligned}
$$

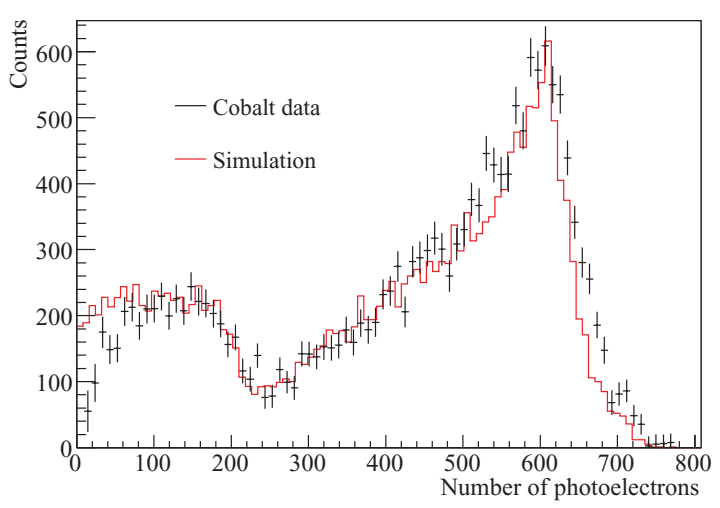

FIG. 3. (Color online) Plot of the ${ }^{57}$ Co spectrum, along with a simulation of the expected spectrum.

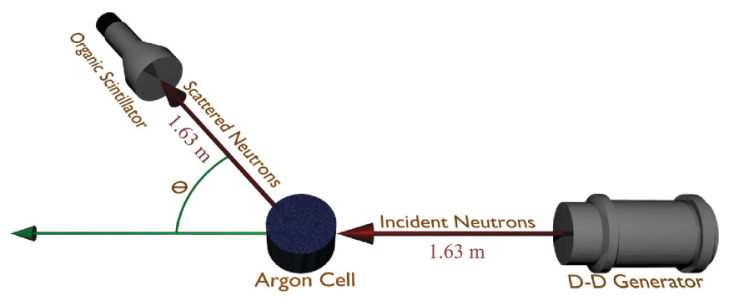

FIG. 4. (Color online) Top view of the neutron scattering setup. Shown are the neutron generator and the organic scintillator. The size of the argon cell is not representative.

where $E_{\text {in }}$ is the incident neutron energy $(2.8 \mathrm{MeV}), A$ is the atomic mass number, and $\theta$ is the scattering angle of the outgoing neutron. Data were taken at 19 different angles corresponding to recoil energies between 10 and $250 \mathrm{keV}$. The setup also included 12 inches of poly between the neutron generator and the organic scintillator to reduce the accidental coincidence rate, although this is not shown in Fig. 4 or 5.

\section{MONTE CARLO SIMULATIONS}

To understand the data, we developed a Monte Carlo simulation of the argon detector, cryostat, organic scintillator, and surrounding laboratory space. The software framework used was RAT, which combines GEANT4 [26], CLHEP [27], and ROOT [28] into a single simulation and analysis package. A detailed optical model of the inner detector and PMTs is included in the Monte Carlo which allows us to estimate smearing of the detected signal. While this model gives results that are in fairly good agreement with our $\gamma$ calibrations, we add an addition smearing term for the neutron scattering analysis to take into account the lower photon yield for a given energy.

An image of the detector geometry can be seen in Fig. 5 . The argon detector is in the central vertical cylinder, where the various layers of steel are set to be semitransparent so that the inner workings are visible. While there was only one organic scintillator detector at a given time in the real experiment, the

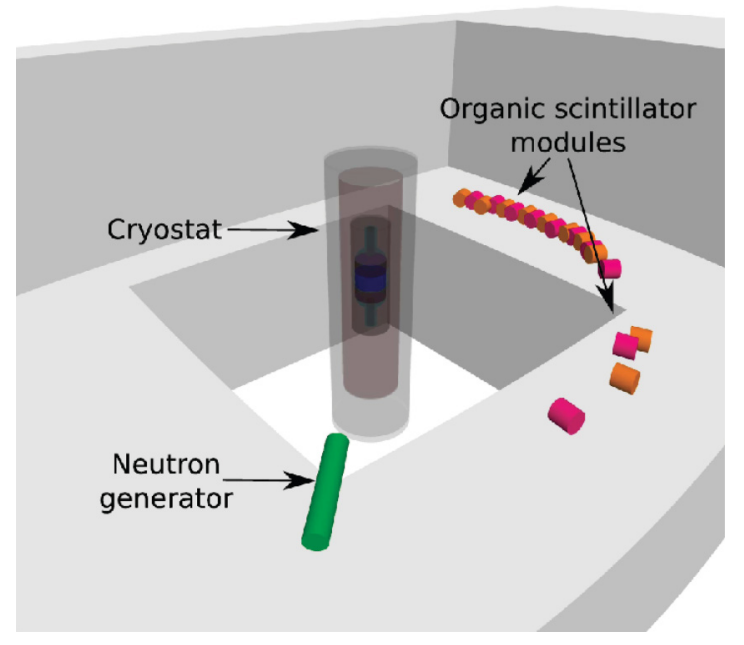

FIG. 5. (Color online) Monte Carlo detector geometry. 
simulation used many such detectors, so as to allow Monte Carlo data for half of the organic scintillator positions to be collected simultaneously and reduce computation time. In the picture, the cylinders off to the right of the argon detector represent the organic scintillator in its various positions. As the adjacent positions of the organic scintillator overlap, independent simulations were performed for each set of colored organic scintillator positions. The 12 inches of poly between the generator and the organic scintillator locations was not included in the simulation.

In addition to the neutron scattering simulation, the detector response to an external ${ }^{57} \mathrm{Co} \gamma$ source was also modeled. The origin of the 122 - and $137-\mathrm{keV} \gamma$ rays was set just outside of the outer vacuum can as in the real detector. The results of this simulation can be seen in Fig. 3 showing very good agreement with the experiment.

\section{ANALYSIS}

The analysis begins by combining the high-gain and lowgain waveforms for each PMT in a given event. To do this the first $2 \mu$ s of each PMT's high-gain and low-gain waveforms are separately averaged to calculate baselines. These baselines are then subtracted to give zero-offset waveforms. Each high-gain waveform is then scanned to determine if the waveform digitizer is saturated. In the case of saturation, the high-gain and low-gain waveforms are aligned and the low-gain waveform's samples are inserted where the high-gain waveform was saturated.

At this point each PMT waveform is scanned to determine the trigger time of the event. The trigger time for each PMT is defined as the time where the waveform reaches $20 \%$ of its maximum height. The average of the two trigger times is taken as the start time for the event and a timing cut is applied to the two PMT waveforms to remove events where the difference in timing is greater than $20 \mathrm{~ns}$. The waveforms from both PMTs are then integrated in two timing intervals, the first from $20 \mathrm{~ns}$ before the trigger time to $100 \mathrm{~ns}$ after and the second from $100 \mathrm{~ns}$ to $5 \mu \mathrm{s}$. The region between 5 and $14 \mu \mathrm{s}$ is scanned for single photoelectron pulses and used to determine the single photoelectron spectrum. Any region where the waveform's voltage value exceeded approximately one-third of a single photoelectron's peak voltage is integrated from $10 \mathrm{~ns}$ preceding the crossover sample to $50 \mathrm{~ns}$ following it. After this procedure has been performed on every event in a run, the run's single photoelectron value is fit and used to convert the integrated waveform charges into photoelectrons.

A PMT asymmetry cut is used to remove events that are near the windows of the detector. The asymmetry is defined as the difference in the signals observed by the two PMTs divided by their sum, and events with an asymmetry of more than $60 \%$ are removed. Events with approximately 2000 photoelectrons or more can cause the saturation of one or both of the detector PMTs, and because this will cause events to have poor energy reconstruction, a cut is applied to remove events in which either PMT's output becomes greater than $2 \mathrm{~V}$. This cut removed nuclear recoil events with energies above $110 \mathrm{keVee}$ for runs with recoil angle below $125^{\circ}$ and nuclear recoil events with

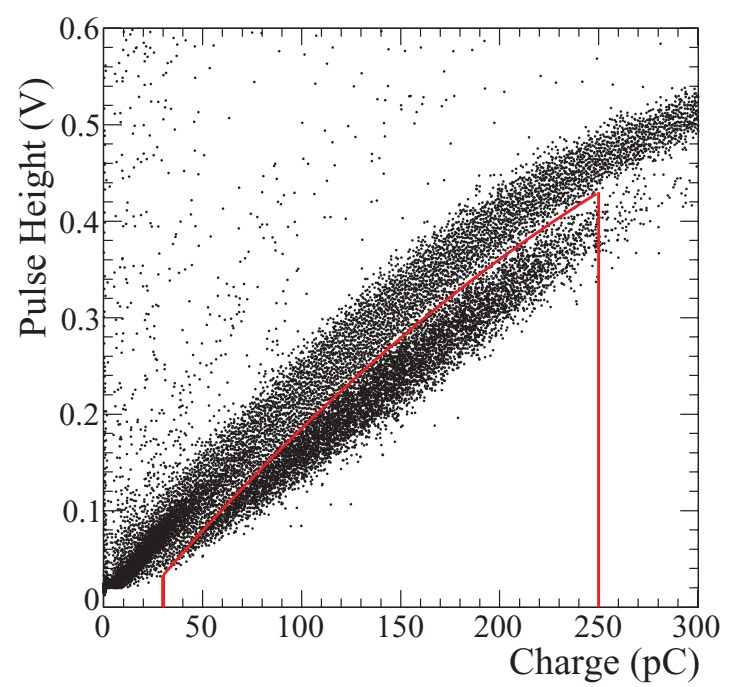

FIG. 6. (Color online) An example of the pulse shape cut applied to the organic scintillator. A scatter plot of the pulse area versus peak pulse height shows a distinct region of neutron events bounded by the red quadratic curve and lines.

energies above $180 \mathrm{keVee}$ for $125^{\circ}$ and $142^{\circ}$ runs. All cuts applied to the data up to this point are considered to be data quality cuts and were applied to both ${ }^{57} \mathrm{Co}$ and neutron runs.

To distinguish neutron scatters from other backgrounds in the neutron data sets, two additional cuts involving the organic scintillator are applied. The first is a time-of-flight (TOF) cut which removes events in which the organic scintillator is triggered before the detector, as well as events in which the neutron arrives late owing to multiple scatters. In addition, this cut also helps remove background $\gamma$ 's from our neutron data sets. The position and width of this cut is set by the location of the single scattering neutrons in the Monte Carlo TOF spectrum.

The second cut associated with the organic scintillator uses the pulse shape in the organic scintillator to distinguish between neutrons and background events. We use a scatter plot of the pulse area within $100 \mathrm{~ns}$ of the organic scintillator trigger versus the organic scintillator waveform's maximum voltage, shown in Fig. 6, to determine a quadratic curve that separates two types of events. This curve divides the scatter plot into two distinct regions: an electronic recoil band that appears for all types of runs and a nuclear recoil band that is present only in neutron runs. The separation of these regions did not appear to change with recoil energy and variation of the regions had a negligible effect on the results.

We apply one final cut to remove electronic recoils from the sample, exploiting the PSD to discriminate between event types in liquid argon. Based on our previous work [10], we define a discrimination parameter, $F_{\text {prompt }}$, as the fraction of light arriving in a prompt time window. For comparison, the mean $F_{\text {prompt }}$ for recoils between 5 and $32 \mathrm{keVee}$ ranges from 0.39 to 0.28 for electronic recoils and 0.56 and 0.7 for nuclear recoils [10]. We apply a relatively loose cut, removing events with $F_{\text {prompt }}<0.35$ from the final sample. We checked the analysis with a tighter cut of $F_{\text {prompt }}<0.50$ and incorporated the differences in the systematic uncertainties. 

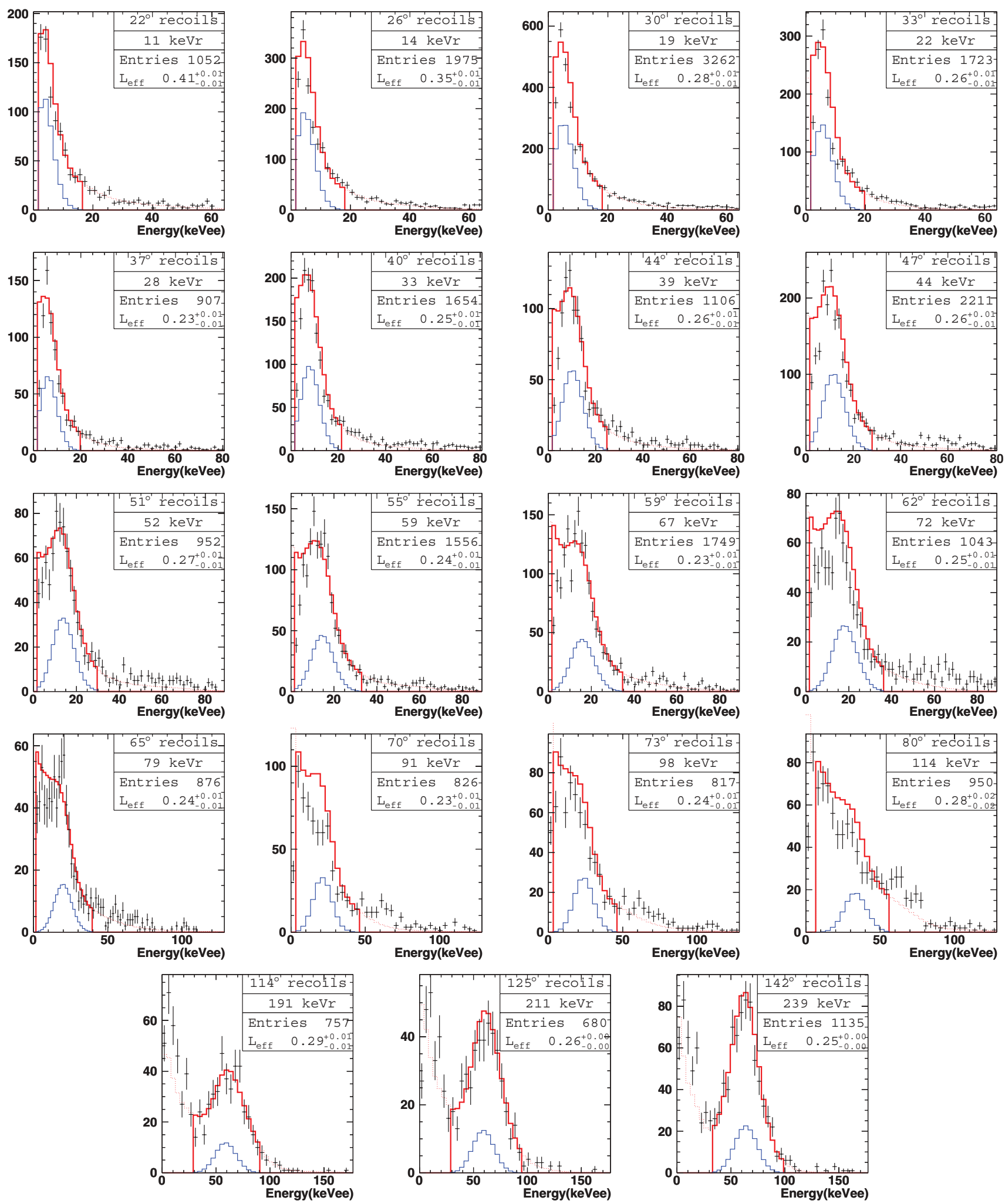

FIG. 7. (Color online) Plotted are the recoil energy spectra for the 19 organic scintillator positions used in this experiment. The data are taken with the organic scintillator located at the angle indicated in the legend of each plot with the corresponding recoil energy indicated just below. The fit value for Leff with the statistical uncertainty from the fit is also listed in the legend of each plot. In each plot, the upper (red) histogram is the output of the GEANT4-based Monte Carlo simulation of single and multiple neutron scatters in the detector. This upper histogram is fit to the data in the solid region, whereas the dotted part shows the MC simulation outside of the fit range. The lower solid histogram (blue) is the subset of the Monte Carlo events where the neutron scatters only once in the detector volume. 
To determine the scintillation efficiency of the liquid argon, the measured energy spectrum for each scattering angle is compared to the energy spectrum produced by the Monte Carlo simulation analyzed with the same asymmetry and TOF cuts. We also use the Monte Carlo simulation to calibrate the TOF cut. For each scattering angle in the Monte Carlo simulation, a TOF spectrum is produced using events that only scattered once in the detector before reaching the organic scintillator. This allows us to find the range of the TOF for single scattering neutrons for each position. After applying the same TOF and PMT asymmetry cut to the simulation as used for the data, a Monte Carlo energy spectrum is then generated for each recoil angle.

There are two convolutions applied to the Monte Carlo recoil spectra before fitting them to the data. First, to account for the variation in the single photoelectron charge, the Monte Carlo photon counts are smeared using the measured single photoelectron charge distribution from the photoelectron calibration data. Second, because the simulations were performed assuming a $100 \%$ scintillation efficiency, an additional smearing of $3.25 \times \sqrt{\left(1-L_{\text {eff }}\right) N_{p e}}$ is applied to the Monte Carlo to account for the difference in counting statistics between a scintillation efficiency of $100 \%$ and that obtained from the data. The proportionality constant of 3.25 empirically accounts for the observed broadness of the clearly resolved peaks at 191-, 211-, and 239-keVr scattering angles. It is well known that noble liquid detectors do not reach the ideal energy resolution predicted by Poisson photoelectron statistics, largely owing to ionization-scintillation anticorrelation $[1,29]$.

The MINUIT fitting package is used to perform a $\chi^{2}$ fit of the Monte Carlo to the data with the normalization and the scintillation efficiency as free variables. Each Monte Carlo spectrum is binned using the same binning as the corresponding recoil data and used to generate a spline for fitting. First, the entire range of the data is used in the fit. Then, a Gaussian is fit to all events in the Monte Carlo identified as singly scattered neutrons. This fit is used to define a new fit range consisting of $\pm 3 \sigma$ around the centroid of the Monte Carlo single-scattered neutron distribution. The final fits are performed over a restricted range around the single-scattering Monte Carlo neutron distribution where we expect to observe our signal. This fitting procedure was checked using the Monte Carlo sample with a set $L_{\text {eff }}$ value of 0.25 and was able to recover this set $L_{\text {eff }}$ at each recoil angle.

The results for all organic scintillator positions are presented in Fig. 7, and the scintillation efficiency as a function of energy is shown in Fig. 8. After studying the systematic effects described in the next section, we found that the individual scintillation efficiency values were constant across the range of recoil energies studied above $20 \mathrm{keVr}$, with a mean of $0.25 \pm 0.01+0.01$ (correlated). However, there still existed substantial differences between the simulation and the data, giving an average $\chi^{2} / \mathrm{DOF}$ (where DOF stands for degree of freedom) of 3.7 for measurements above $20 \mathrm{keVr}$. This disagreement is addressed in the next section. Below $20 \mathrm{keVr}$, our data exhibit an upturn in scintillation efficiency as the energy goes to zero, and we were unable to find an experimental cause for this upturn. It is therefore unknown if this is a physically real effect or if we lose our ability to

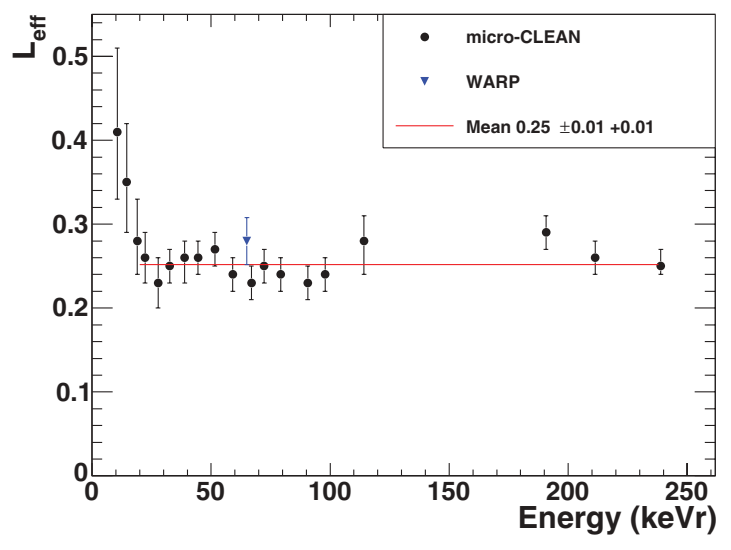

FIG. 8. (Color online) Scintillation efficiency as a function of energy from 10 to $250 \mathrm{keVr}$. The weighted mean (red line) is generated from the data above $20 \mathrm{keVr}$ and puts the mean scintillation efficiency at 0.25 . The value measured by WARP is 0.28 at $65 \mathrm{keVr}$ [3].

distinguish nuclear recoils from other backgrounds at these low energies. All observed values and uncertainties are listed in Table I.

\section{SYSTEMATIC UNCERTAINTIES}

The sources of uncertainty considered are categorized as those associated with detector operation, triggering effects, Monte Carlo background normalization, TOF window, and fit range effects. The uncertainties from the sources discussed in this section are all combined and the final resulting uncertainty for each scattering angle can be found in Table I.

TABLE I. Table of energies and scintillation efficiencies from Fig. 8. $L_{\text {eff }}$ values for energies above $20 \mathrm{keVr}$ also have an additional correlated error of +0.01 . The uncertainties shown are the combined statistical and systematic uncertainties.

\begin{tabular}{lcccc}
\hline \hline Scattering angle (deg) & Energy (keVr) & $L_{\text {eff }}$ & + & - \\
\hline 22 & 11 & 0.41 & 0.10 & 0.08 \\
26 & 15 & 0.35 & 0.07 & 0.06 \\
30 & 19 & 0.28 & 0.05 & 0.04 \\
33 & 22 & 0.26 & 0.03 & 0.03 \\
37 & 28 & 0.23 & 0.03 & 0.03 \\
40 & 33 & 0.25 & 0.02 & 0.02 \\
44 & 39 & 0.26 & 0.02 & 0.03 \\
47 & 45 & 0.26 & 0.02 & 0.02 \\
51 & 52 & 0.27 & 0.02 & 0.02 \\
55 & 59 & 0.24 & 0.02 & 0.02 \\
59 & 67 & 0.23 & 0.02 & 0.02 \\
62 & 72 & 0.25 & 0.02 & 0.02 \\
65 & 79 & 0.24 & 0.02 & 0.02 \\
70 & 91 & 0.23 & 0.02 & 0.02 \\
73 & 98 & 0.24 & 0.02 & 0.02 \\
80 & 114 & 0.28 & 0.03 & 0.04 \\
114 & 191 & 0.29 & 0.02 & 0.02 \\
125 & 211 & 0.26 & 0.02 & 0.02 \\
142 & 239 & 0.25 & 0.02 & 0.01 \\
\hline \hline
\end{tabular}


The first group of considered uncertainties deals with the data taking and stability of the neutron and ${ }^{57}$ Co data runs. Because the ${ }^{57} \mathrm{Co}$ runs are used to calibrate the light yield of the detector, the fit error of the ${ }^{57} \mathrm{Co}$ peak and its stability over time directly affect the measured scintillation efficiency. These are estimated to be $2 \%$ and $1.6 \%$, respectively. There is a second uncertainty in the angle of the organic scintillator relative to the neutron generator that in turn introduces an uncertainty in the corresponding energy via Eq. (1). We have determined the uncertainty of the angular position of the organic scintillator to be $1.3^{\circ}$ at each position.

We examined the effects of the trigger efficiency, specifically looking to address the upturn observed at low energies which could be explained by a bias introduced by the trigger level. We took data for the $22^{\circ}$ run at three different hardware triggers and the $26^{\circ}$ run at two different hardware triggers, and we examined the effect of hardware and software triggers on both the asymmetry cut and the final scintillation efficiency values. In all cases, the scintillation efficiency distributions did not systematically change by varying the cuts and hardware threshold. We also performed a toy Monte Carlo using the time dependence of the scintillation light [10] and the observed single photoelectron distribution to estimate possible threshold effects. This study found the effect of any threshold bias given our hardware trigger level to be less than $1 \%$, much smaller than the other errors in the measurement. Therefore, we conclude that the triggering threshold does not explain the upturn at low energies.

A third source of uncertainty arises because the Monte Carlo simulation, as mentioned at the end of Sec. V, does not exactly reproduce the observed background shape. This can be seen by comparing the dashed and solid red lines in the large angle scattering spectra of Fig. 7. To account for this inconsistency, the data for each recoil energy were reanalyzed under the assumption that the size of the multiple scattering background in the histograms used to perform the fits varied by $\pm 50 \%$ relative to that predicted by the Monte Carlo. The variations observed in this reanalysis are approximately $10 \%$ below $20 \mathrm{keVr}$ and $2 \%$ above $20 \mathrm{keVr}$ and are included in the errors listed in Table I.

To determine the uncertainty owing to the TOF cuts, the TOF window was expanded separately up and down in time by $50 \%$. This allows for recoil neutrons with smaller TOFs to be included when the window is expanded downward and larger TOF neutrons when expanded upward. The effect of this variation was mostly in the lowest three data points and allowed them to move downward in scintillation efficiency by about 0.04 .

There is an uncertainty associated with fitting the data in a limited range around the predicted single-scattered neutron peak position. To estimate this uncertainty, we expand the fit range to include $\pm 5 \sigma$ around the centroid of the singlescattered neutrons, instead of the $3 \sigma$ range used in the standard fit. The result of the wider fit range is to systematically push the determined scintillation efficiency up for energies between 20 and $120 \mathrm{keVr}$. This effect appears to be caused by a disagreement in the high-energy tails of the data and Monte Carlo, similar to the disagreement observed between Monte Carlo and data at lower energies for the high recoil angles. Changing the fit range adds a correlated error of +0.01 to the measured mean scintillation efficiency in Fig. 8.

\section{RESULTS}

The scintillation efficiency for nuclear recoils in liquid argon has been measured relative to electronic recoils for nuclear recoil energies from 10 to $250 \mathrm{keVr}$. The scintillation efficiency values found at each of the recoil angles can be found in Table I and are plotted in Fig. 8. The ratio of the nuclear recoil scintillation response to the electronic recoil response is $0.25 \pm 0.01+0.01$ (correlated) for recoils above $20 \mathrm{keVr}$. An observed upturn in the scintillation efficiency below $20 \mathrm{keVr}$ is currently unexplained. The scintillation efficiency for nuclear recoils should also have some dependence on any applied electric fields and this is a topic for further research.

\section{ACKNOWLEDGMENTS}

Computer resources were supplied by Yale University Biomedical High Performance Computing Center, NIH Grant No. RR19895, and in part by the facilities and staff of the Yale University Faculty of Arts and Sciences High Performance Computing Center. We are also grateful for the support of the US Department of Energy. This work was supported by the David and Lucille Packard Foundation.
[1] E. Aprile et al., New Astron. Rev. 49, 289 (2005)

[2] D. B. Cline et al., Nucl. Phys. B, Proc. Suppl. 124, 229 (2003).

[3] R. Brunetti et al., New Astron. Rev. 49, 265 (2005).

[4] P. Benetti et al., Astropart. Phys. 28, 495 (2008).

[5] A. Rubbia, J. Phys.: Conf. Ser. 39, 129 (2006).

[6] G. Jungman, M. Kamionkowski, and K. Griest, Phys. Rep. 267, 195 (1996).

[7] Z. Ahmed et al. (CDMS), Science 327 (2010).

[8] J. Angle et al., Phys. Rev. Lett. 100, 021303 (1983).

[9] A. Hitachi, T. Takahashi, N. Funayama, K. Masuda, J. Kikuchi, and T. Doke, Phys. Rev. B 27, 5279 (1983).
[10] W. H. Lippincott, K. J. Coakley, D. Gastler, A. Hime, E. Kearns, D. N. McKinsey, J. A. Nikkel, and L. C. Stonehill, Phys. Rev. C 78, 035801 (2008).

[11] T. Takahashi, S. Konno, T. Hamada, M. Miyajima, S. Kubota, S. Nakamoto, A. Hitachi, E. Shibamura, and T. Doke, Phys. Rev. A 12, 1771 (1975).

[12] M. Miyajima, T. Takahashi, S. Konno, T. Hamada, S. Kubota, H. Shibamura, and T. Doke, Phys. Rev. A 9, 1438 (1974).

[13] T. Doke, A. Hitachi, J. Kikuchi, K. Masuda, H. Okada, and E. Shibamura, Jpn. J. Appl. Phys. 41, 1538 (2002).

[14] J. Lindhard, M. Scharff, and H. Schiøtt, Mat.-Fys. Medd. - K. Dan. Vidensk. Selsk. 33, 1 (1963). 
[15] P. Sorensen et al., Nucl. Instrum. Methods Phys. Res., Sect. A 601, 339 (2009).

[16] E. Aprile et al., Phys. Rev. C 79, 045807 (2009).

[17] E. Aprile, K. L. Giboni, P. Majewski, K. Ni, M. Yamashita, R. Hasty, A. Manzur, and D. N. McKinsey, Phys. Rev. D 72, 072006 (2005).

[18] V. Chepel, V. Solovov, F. Neves, A. Pereira, P. J. Mendes, C. P. Silva, A. Lindote, J. Pinto da Cunha, M. I. Lopes, and S. Kossionides, Astropart. Phys. 26, 58 (2006).

[19] A. Manzur, A. Curioni, L. Kastens, D. McKinsey, K. Ni, and T. Wongjirad, Phys. Rev. C 81, 025808 (2010).

[20] A. Hitachi, T. Doke, and A. Mozumder, Phys. Rev. B 46, 11463 (1992).

[21] D.-M. Mei, Z. Yin, L. Stonehill, and A. Hime, Astropart. Phys. 30, 12 (2008).
[22] A. Hitachi, T. Takahashi, T. Hamada, E. Shibamura, N. Funayama, K. Masuda, J. Kikuchi, and T. Doke, Nucl. Instrum. Methods 196, 97 (1982).

[23] C. Regenfus, IDM2006 Proc., World Sci. 15, 32 (2007).

[24] O. Cheshnovsky, B. Raz, and J. Jortner, J. Chem. Phys. 57, 4628 (1972).

[25] D. N. McKinsey, C. R. Brome, J. S. Butterworth, R. Golub, K. Habicht, P. R. Huffman, S. K. Lamoreaux, C. E. H. Mattoni, and J. M. Doyle, Nucl. Instrum. Methods Phys. Res., Sect. B 132, 351 (1997).

[26] http://geant4.cern.ch/.

[27] http://proj-clhep.web.cern.ch/.

[28] http://root.cern.ch/.

[29] E. Conti et al., Phys. Rev. B 68, 054201 (2003). 